This paper will appear in Mertens, K. \& Guenzler, I. (eds.): Wahrnehmen, Fühlen, Handeln. Phänomenologie im Widerstreit der Methoden. Paderborn: Mentis 2013.

As always, comments are very welcome!

\title{
How Essential are Essential Laws? A Thought Experiment on Physical Things and Their Givenness in Adumbrations
}

\author{
Harald A. Wiltsche \\ University of Toronto, Canada/University of Graz, Austria \\ harald.wiltsche@uni-graz.at
}

Surprisingly enough, the amount of literature on thought experiments (hereinafter referred to as TEs) increased dramatically only within the last two decades. This is surprising because TEs are an integral part of our epistemic practices in virtually all segments of human endeavour. Suppose that you are unsure whether the blue shirt fits the yellow pants. What do you do? You imagine yourself wearing this specific combination. What did Galileo do to refute the Aristotelian law of falling bodies and to establish his own law of free fall? He imagined throwing things from a tower. What did Frank Jackson do to show that physicalism is wrong? He imagined Mary.

Within philosophy, TEs seem to be mainly utilized by philosophers from the analytical camp. There, TEs are a widely accepted tool for the analysis of a huge variety of philosophical problems. ${ }^{1}$ However, so far, no systematic attempts to analyze TEs from a phenomenological perspective have been made. ${ }^{2}$ This paper is intended as a step towards filling this gap. Although I will only deal with one single philosophical TE, I hope to give at least some insight into the method of thought experimental reasoning. Yet, in doing so, I not only wish to shed some light on the prospects and limits of TEs. I also hope to contribute to a better understanding of phenomenology, namely of the eidetic method in general and to the notion of essential laws in particular.

I will proceed as follows: Before I can focus on my actual subject matter, I must to do some stage-setting in the first section. I will characterize the notion of (givenness in) adumbrations and its consequences for the phenomenology of perception. I will then go on to say a word or two on the concepts of essentiality and apodictic Evidenz. Section two is concerned with the presentation of a concrete TE, namely the eyeball-TE. This TE seems to undermine the phenomenological position according to which givenness in adumbrations belongs to the very essence of physical thinghood. Section three is concerned with two tasks: On the one hand, I will give a general characterization of what I take TEs to be. On the other hand, I shall explain why the eyeball-TE fails in undermining the phenomenological position. Crucial for my argument is the distinction between first-person-imagination and second-personimagination. However, as I will emphasize in section four, the eyeball-TE not only deserves criticism. It also delimits the scope of the phenomenological notion of apodicticity. Finally, in section five, I will briefly reflect on the epistemological significance of the distinction between first- and second-person-imagination.

\footnotetext{
${ }^{1}$ This is not to say, however, that there aren't analytical philosophers who find fault with the method of TEs. Cf. e.g. Wilkes 1988; Dennett 1991, ch. 14; Weinberg, Nichols \& Stich 2001.

${ }^{2}$ To the best of my knowledge, there are only three phenomenological contributions to the debate on TEs so far: Mohanty 1991; Froese \& Gallagher 2010; Fehige \& Wiltsche 2012 (forthcoming).
} 


\section{Adumbrations, Essential Laws and Apodicticity}

In his recent book on the philosophy of perception Walter Hopp writes:

"Anything that can be observed from more than a single point of view, as material objects can, also has more parts and properties that can be presented in a single perceptual act. Perception is always inadequate to its object, and this inadequacy is itself a phenomenologically discoverable feature of such an experience; in the face of an external object we 'know' that there is more to it than what is strictly revealed [...]. This stems, not from any peculiar deficiency on our part, but from the essence of spatial objects." (Hopp 2011, 54-55; emphasis added)

Or, as Aron Gurwitsch put it almost 50 years ago:

"No perceptual appearance of a material thing can be altogether devoid of references to aspects other than that actualized at the moment. To assume that as possible would entail the consequence of a material thing perceptually apprehended through a single perception. [It would lead to the absurd admission] that, at least under certain circumstances, a thing might coincide with one of its perceptual appearances." (Gurwitsch 2010, 229; emphases added)

Both Gurwitsch and Hopp refer to an issue that is widely discussed not only, ${ }^{3}$ but especially in the phenomenology of perception. Both refer to the fact that physical things are always and necessarily given inadequately, or, as Husserl sometimes puts it, given in adumbrations. Let me illustrate what this means (cf. also Husserl 1983, §41-42; Husserl 1997, ch. 3).

Imagine perceiving a red mug. Obviously, the mug belongs to the category "physical thing". With regard to this categorial membership, however, many of the mug's properties are irrelevant. You could imagine the mug being blue without violating the respective categorial boundaries. You could imagine the mug being a perfect sphere and it would still belong to the category "physical thing". You could even imagine the mug floating away from the ground without the application of external force and it still would be an instantiation of the category "physical thing".

While many properties are open to arbitrary variation in this sense, other properties seem to be of a different nature. Take the aforementioned fact that all members of the category "physical thing" are given in adumbrations. What I mean is this: Usually, while being intentionally directed towards a physical thing, you are directed to the thing in its entirety. You simply perceive the mug. On closer inspection, however, there is a fundamental discrepancy between your intention and its experiential fulfillment: All that is given perceptually at a particular point in time is just one single profile of the thing, namely its front side. To be sure, you can change your position and make the back side the front side, and vice versa. Yet, this does not change the fact that you are still directed towards a thing that is given perspectively. When it comes to physical things, you simply can't have only front sides without any back sides. Physical things are always and necessarily given in partial aspects. That is what Husserl calls "givenness in adumbrations". ${ }^{4}$

\footnotetext{
${ }^{3}$ To be sure, the phenomenon I am about to describe is not only discussed in the phenomenological literature. Take for instance Peter Strawson's approving portrayal of A. J. Ayer's philosophy of perception: “According to Ayer, the starting point of serious thought on the matter of perception consists in the fact that our normal perceptual judgements always 'go beyond' the sensible experience that gives rise to them; for those judgements carry implications which would not be carried by any 'strict account' of that experience." (Strawson 2002, 91)

${ }^{4}$ Actually, this is not quite true. Husserl not only uses the notion "adumbration" for a genuinely perspectival phenomenon. Quite generally, he speaks of "adumbrations of color, of smoothness, of shape, etc." (Husserl 1983, 88).
} 
However, crucial to Husserl's position is that we are not talking about empirical matters of fact here. Husserl rather claims that "[i]t is neither an accident of the own peculiar sense of the physical thing nor a contingency of our 'human constitution,' that 'our' perception can arrive at physical things themselves only through mere adumbrations of them" (Husserl $1983,90-91)$. One way to justify this claim is this: If no external force is applied, all physical things tend to fall downward, towards the center of the earth. You will have a hard time finding an object that does not conform to this law in the normal course of events. But, as I have said, it is easy to imagine physical things floating away from the ground without thereby transgressing the categorial boundaries of physical thinghood. It is for this reason that all nomological constraints as well as all empirical matters of fact in general are said to be contingent: They are what they are. But we could imagine them fundamentally different. Apparently, our imaginative capacities open up a route to a sphere of possibilities that is substantially richer than the sphere of nomological possibilities.

But now imagine producing a series of arbitrary variations of physical things. Furthermore, in imagining these variations, try to deliberately "think away" all connections to actual or past experiences and thus free your imagination from your natural expectations towards reality. Finally, do not bother with questions of realizability; let your imagination go wild and accept all variations that occur to you, no matter how divorced from reality they may be. The point is that even if you follow these instructions, all variations still share a common core of invariant structures. As long as we do not transgress the boundaries of the category "physical thing" (and, for instance, imagine a geometrical object), all variants are given, for instance, in spatial adumbrations. To single out and directly intuit this invariant structure is the notorious "seeing of an essential law". In doing so, we directly grasp "a universal essence which [...] prescribes an intellectually seen generical rule for every particular object becoming intended to in multiplicities of concrete mental processes" (Husserl 1983, 341).

But there is more: Once you think you have unveiled an essential law, it is natural to put the respective law to the test by looking for counterexamples. The essential law of givenness in adumbrations, for instance, would be falsified by the imagination of a solid physical thing that has no back side at all, i.e. that is not given in adumbrations. Try to imagine such a thing. You will notice that you fail, no matter how hard you try. But, remarkably, even that is not all. It is not just impossible to imagine a solid physical thing without a back side of some sort. What is more, whenever you try to imagine a thing like that, you end up with an object of the kind you initially wanted to avoid: you end up with a physical object that is given in adumbrations. Let me put this fairly important point differently: With regard to the property of givenness in adumbrations, it is not only impossible to imagine a concrete counterexample. Every attempt to do so also confronts you with just another validating instance of the initial object in question. ${ }^{5}$ This corresponds to the phenomenological notion of apodicticity: Something is apodictically evident "if it cannot be imagined as nonexistent without being presupposed as existing in the same act of thinking" (Schmid 2001, 225). Or, to put it in the words of David Woodruff Smith: "[A]n evident experience is apodictic if I cannot doubt the existence and properties of the object presented in the experience, I cannot imagine their non-being (in the face of this experience)." (Smith 2007, 335)

\footnotetext{
${ }^{5}$ From now on, I will distinguish two notions of unimaginability: On the one hand, „strong unimaginability" applies to cases in which $\mathrm{x}$ is not just unimaginable, but in which the attempt to imagine the non-being of $x$ results in the recurrent givenness of $x$. On the other hand, to imagine an icosidodecahedron is impossible simpliciter, i.e. impossible without confronting us with any contradictory results.

${ }^{6}$ Here is how Husserl himself puts it: "An apodictic evidence [...] is not merely certainty of the affairs or affair-complexes (states-of-affairs) evident in it; rather it discloses itself, to a critical reflection, as having the signal peculiarity of being at the same time the absolute unimaginableness [...] of their non-being, and thus excluding in advance every doubt as 'objectless,' empty. Furthermore the evidence of that critical reflection likewise has the dignity of being apodictic, as does therefore the
} 
To sum up: Phenomenologically construed, the statement that all physical things are necessarily given in adumbrations is an essential, non-empirical law that is apodictically evident. It is apodictically evident because the nonexistence of the necessitation relation between the properties denoted by the predicates "being a physical thing" and "given in adumbrations" is unimaginable in a strong sense of the term. And it is non-empirical because it seems to hold necessarily, i.e. in any possible world.

\section{On Mad Scientists and Eyeball Surgeries}

Given the strength of the phenomenological position, a number of possible criticisms instantly come to mind. For instance, one could question whether imaginability indeed is a reliable guide to possibility, impossibility and necessity. ${ }^{7}$ After all, we are unable to imagine, say, an icosidodecahedron without thereby being committed to claims about its modal status. The focus in this paper, however, is on a different line of critique. As I have mentioned earlier, I will restrict myself to a particular TE that seems to prove the untenability of the position that I have characterized so far.

Remember how the claim that the givenness of adumbrations belongs to the very essence of physical thinghood is justified: However many variants of physical things you may imagine, all of them share the common structure of givenness in adumbrations. Additionally, in trying to imagine its non-being, you either transgress the categorial boundaries of physical thinghood or you end up with the very being of the structure in question. Hence, the truth of the statement that perspectivity belongs to the essence of physical thinghood is said to be apodictically evident. Let us suppose that we accept all this. This act of acceptance expresses our trust in our imaginative capacities - after all, it is these capacities that justify the apodicticity-claim in the first place. But now suppose that the same imaginative capacities confronted us with a situation in which the essential law of perspectivity was not apodictically evident. Consider the following TE that seems to involve a scenario of exactly this kind. ${ }^{8}$

Imagine perceiving one of the miniature globes that people like to put on their desks. First of all, since a globe is a physical object, it is given in adumbrations. Secondly, we know a couple of things about globes. We know, for instance, where Toronto is. And we know that, if we go a little bit further to the East, we will find Montreal, then Halifax, then the Atlantic Ocean, then Spain, then France, and so on and so on. Ultimately, after doing one full turn, we know that we will arrive in Toronto again. But now imagine running into a mad scientist ${ }^{9}$ who is specialized in eyeball-surgeries and who decides to do a little experiment on you. First, he takes your two eyeballs out. Then he constructs a sphere of, say, hundred eyeballs. The sphere is wide enough to be completely wrapped around the globe. Finally, the hundred eyeballs are connected to your brain. It may take you some time to adapt and to blend the information of the hundred eyeballs together. But the point should be obvious: Since it is completely covered with eyeballs, you perceive the globe from all perspectives at once. No complete turn is needed anymore to do the trip from Toronto to Toronto. Everything is given at one full blow.

Before I enter a more detailed analysis of this TE, two things should be noted right away: First, it seems apparent that the TE refutes both phenomenological claims at once. The TE confronts us with a situation in which a physical thing seems to be given perceptually, but

\footnotetext{
evidence of unimaginableness of what is presented with <apodictically> evident certainty. And the same is true of every critical reflection at a higher level." (Husserl 1960, pp. 15-16)

${ }^{7}$ Cf. for an overview of this discussion as it takes place within analytical philosophy: Gendler \& Hawthorne 2002.

${ }^{8}$ It must be noted that this TE is not my invention. A slightly different version originally stems from Jeff Hilderley.

${ }^{9}$ Unfortunately, I am unable to further pursue the question as to why the thought experimental universe is mostly populated by scientists who are mad, evil or both.
} 
not in adumbrations. It thus falsifies the claim that the givenness in adumbrations belongs to the essence of physical thinghood. Yet, if the TE is successful in showing this much, it also falsifies the claim that a physical thing, which is not given in adumbrations, is unimaginable in a strict sense of the term. Hence, the TE also falsifies the claim that the law of givenness in adumbrations is apodictically evident.

Secondly, the thought experiment seems to undermine the difference between essences and empirical generalities, a difference which is of utmost importance for the entire phenomenological project. Remember the claim that empirical knowledge is contingent. It is contingent insofar as we could imagine a world in which any facet of empirical reality is fundamentally different. Contrariwise, essential laws are characterized by their uniformity through changing imaginative variations. Even if we free ourselves from our natural expectations towards reality, no world is imaginable in which an essential law does not hold. But now, the eyeball-TE seems to confront us with such a world. It confronts us with a world in which a mad scientist makes us perceive physical things that are not given in adumbrations. As we have seen earlier, Husserl claims that it is not "a contingency of our 'human constitution,' that 'our' perception can arrive at physical things themselves only through mere adumbrations of them" (Husserl 1983, 90-91). This claim seems to be refuted by the eyeball-TE. The TE seems to prove that the givenness in adumbrations is just a matter of our physiological make-up.

\section{Why the TE Fails: First-Perspective Imagination and Third-Perspective Imagination}

As I have mentioned earlier, the eyeball-TE suffers from several flaws. But let me, before I go into details, say a word or two on the general nature of TEs. In performing TEs we describe an imaginary scenario in order to evoke certain intuitions about what would happen if the described scenario was actually to obtain (cf. Gendler 2000, 34). We do this, however, for a special purpose: A TE is an experiment precisely because it is used to corroborate or falsify a particular target thesis. Thus, following a similar characterization offered by Sören Häggqvist $(2009,63), I$ take it that this model covers the core structure of many TEs, including the eyeball-TE: ${ }^{10}$

$$
\begin{aligned}
& T \\
& \diamond C \\
& T \supset(C \square \rightarrow W) \\
& C \square \rightarrow \neg W \\
& \neg T
\end{aligned}
$$

Even though it may not always be expressed explicitly, the starting point usually is the target thesis $T$. The second step, as stated in premise 2, is the description of a counterfactual scenario $C$. However, it is crucial for the quality of a TE that $C$ is not just any scenario. $C$ has to be possible in some relevant sense in order for the thought experiment to be epistemically fruitful. Hence the modal claim that $C$ is possible. Premise 3 states that, according to $T$, we are committed to the truth of a statement $W$ in scenario $C$. However, this is exactly what the thought experimenter opposes: Premise 4 states that $W$ is false in $C$. Hence, the conclusion that $T$ is false.

Following this model, the eyeball- TE may be recast as follows:

I) It is apodictically evident and thus an essential law that all solid physical things are given in adumbrations.

II) A globe is a solid physical thing.

\footnotetext{
${ }^{10}$ The obvious problem with this model is that it only applies to destructive TEs. It is therefore unable to cover constructive TEs such as Stevin's proof of the law of balanced forces on inclined planes.
} 
III) It is possible that we could be $\mathrm{x}$, where $\mathrm{x}$ is a subject that perceives with a sphere of hundred eyeballs.

IV) Under the condition that I) is true, it follows necessarily that, if we were $x$, a globe would be given in adumbrations.

V) But, evidently, if we were $x$, a globe would not be given in adumbrations.

VI) We are under the same realizable conditions like $x$ in all relevant aspects. ${ }^{11}$

VII) Therefore, I) is false.

The benefit of reconstructions such as these is to make the likely points of criticism more obvious. For instance, one of the first things to notice is this: Quite generally, the weak spot of many TEs are the counterfactual scenarios that they involve. These scenarios may be said to be impossible in some relevant sense of the term, or they may be said to be inadequate with respect to the respective target thesis. In our current case, however, both of these strategies are not very promising: Given our current medical resources, a surgery of the kind imagined in the TE may be technologically impossible. Yet, there doesn't seem to be any deeper reason to deny that future generations of mad scientists will be able to perform procedures like this. Additionally, it is beyond doubt that a modification of the empirical conditions of our vision is relevant for our perceptual and imaginative capacities. Hence, it seems pointless to discard the whole TE solely on the grounds of the modal claim that is associated with III).

Yet, there is another aspect that is noteworthy: Take a closer look at the target thesis. It is the statement that it is apodictically evident and thus an essential law that all solid physical things are given in adumbrations. However, what does it exactly mean to say that the respective law is apodictically evident? As we have seen earlier, it means that it is unimaginable in a strong sense to imagine an object that both belongs to the category "physical thing" and is not given in adumbrations. But now note the following: The statement that such-and-such is unimaginable is fundamental in the sense that we simply report what in fact isn't imaginable, given our current imaginative capacities. In a way, the status of this statement is that of a protocol sentence (no further historical connotations intended!): We are simply reporting the experiences that we have when we use our imaginative capacities to reflect on possible modes of the givenness of physical things.

Let us, following a suggestion of Zeno Vendler (1984, ch. 3), call the kind of imagination that is at play here first-person-imagination. Here is an example showing what this means: Suppose that you are standing on top of a cliff. And suppose furthermore that you imagine yourself swimming in the ocean below you. One way to do this is to imagine the experiences that you would have if you were in the respective situation. From the perspective of firstperson-imagination, you would imagine having experiences such as "the cold, the salty taste, the rug of the current, and so forth" (Vendler 1984, 43).

Third-person-imagination, on the other hand, is to imagine oneself from a particular external perspective. To do so is not at all different from a representation of other persons. Standing on top of the cliff, I could imagine myself swimming down there in the water. However, it would be equally easy to imagine Dana, Lina, Valentin, or a complete stranger swimming

\footnotetext{
${ }^{11}$ This additional premise blocks another possible reaction to the TE. Suppose that one accepts the TE as far as to premise V). In this case there are two different options to go on from here: The first option is to overthrow I). The second option is to stick to $\mathrm{I}$ ), but to deny that $\mathrm{x}$ actually perceives a solid physical thing. However, $\mathrm{I}$ find the latter strategy highly questionable. Imagine that the mad scientist does his surgery in a split-second without you even noticing it. What would it mean to deny that $x$ perceives a physical thing in this situation? It would mean that the thing changed its categorial status from one split second to the other. Additionally, it would mean that the givenness in spatial adumbrations is the only criterion by which physical things and non-physical things are differentiated. Either of these consequences is highly counterintuitive.
} 
there. While first-person-imaginations are about the experiences one would have in a particular situation, third-person-imaginations merely give an external view on the respective situation.

The distinction between first-person-imagination and third-person-imagination is crucial if we want to get a clearer view on the eyeball-TE. Take a closer look at what the TE invites us to do: It invites us to imagine that we no longer perceive with two, but with hundred eyeballs that are spherically arranged. As I have pointed out, there is nothing wrong with this imaginary scenario or with the modal claim associated with it. However, what is it that we actually do when we imagine this scenario? Are we imagining the experiences that we would have if we were in the respective situation? Obviously, this is not the case. To imagine being a subject that perceives with a sphere of hundred eyeballs is something completely different than to imagine the experiences such a subject would have. While the former merely requires the perspective of third-person imagination, the latter requires the perspective of first-person imagination.

With these considerations as a backdrop, we are now in a position to see what is wrong with the eyeball-TE: On a phenomenological view, the essential law that physical things are necessarily given in adumbrations is apodictically evident because a counter-case is unimaginable in a strong sense of the term. Consequently, a destructive TE would only be successful if it confronted us with the experience of a physical thing that is not given in adumbrations. But, obviously, this is not what the experiment does. Even if we accept all of its premises, it is simply not the case that the TE somehow affects the experiences that, on a phenomenological view, justify the respective essential law in the first place. We may be able to imagine that we (or someone else, for that matter) perceive with a sphere of hundred eyeballs. But, evidently, this has no implications for our ability to imagine concrete variations of physical things from a first-person-viewpoint.

\section{Why the TE is (Partly) Successful: Apodicticity and Fallibility}

Let me briefly summarize the results of the previous section: TEs employ imaginary scenarios in order to evoke certain intuitions about what would happen if the described scenario was actually to obtain. To be sure, the eyeball-TE does evoke certain intuitions; it evokes intuitions about the possibility of a modification of our visual apparatus. Yet, the problem is that these intuitions are not on the same level and thus do not conflict with the intuitions that justify the target thesis. It is for this reason that the TE fails in undermining the phenomenological position, both with regard to the essential law of givenness in adumbrations and with regard to the apodicticity-claim that goes along with it.

But is that all there is to say about the eyeball-TE? By no means. As I have indicated in my introductory remarks, the TE not only deserves criticism. If it is understood correctly, it helps to clarify the phenomenological concept of apodicticity. Let us turn to that now.

Remember my claim that the eyeball-TE makes use of what I have called third-personimagination. This is to say that the TE does not directly oppose the claim that we imagine such-and-such and aren't able to do otherwise. The TE rather invites us to imagine that our perceptual apparatus is modified. As we have seen in the previous section, the possibility of this scenario neither affects our actual imaginative capacities, nor our actual stream of imaginative experiences. And it is for this reason that the TE fails, at least in its intended sense. However, on the other hand, it is also clear that this negative result is independent from the modal status of the scenario of an eyeball-surgery. This is to say that even though the TE fails in its initial sense, a surgery of the kind imagined in the TE still has to be regarded as a possibility. But if this is admitted, we also have to agree that a more or less substantial modification of our stream of experiences cannot be ruled out in principle. We may not be 
able to say precisely what would happen if we perceived with a sphere of hundred eyeballs. But it would be dogmatic to deny that our experiences were likely to undergo a fundamental change.

Yet, isn't this enough to severely damage the phenomenological position, at least when it comes to the claim that the essential law of givenness in adumbrations is apodictically evident? The problem is this: The apodicticity-claim concerning the respective law is justified by the factual experience of strong unimaginability of physical things that are not given in adumbrations. However, if the previous considerations are sound, the eyeball-TE shows that a fundamental change of our stream of experiences has to be regarded as a possibility. We therefore also have to admit that, in principle, there could be subjects for whom the initial apodicticity-claim is falsified by the absence of the experience of strong unimaginability. Hence, we have to conclude that the law of givenness in adumbrations may be apodictically evident for us, here and now. But it is obviously not apodictically evident that it will be that way forever. Thus, the eyeball-TE seems to do its job after all: It seems to show that a situation is possible in which the initial apodicticity-claim is in fact falsified.

Let me, in order to clarify what is at stake here, start with a question that may strike you as rather odd: What is the problem with a claim that is said to be both apodictically evident and falsifiable? The answer is that from a traditional perspective, a claim is apodictically true if and only if it is absolutely certain and thus has total security from error. Such a claim would be context-irrelative in a strong sense of the word: A claim has total security from error if and only if no scenario is possible in which the respective claim could be false. Thus, with some notable exceptions such as Descartes' cogito, apodictic truth is mostly reserved for tautological formulae. Let us call this conception "apodicticity" ${ }_{1}$.

If phenomenology really relied on apodicticity ${ }_{1}$, the eyeball-TE indeed would be a successful refutation of the initial apodicticity-claim. The TE shows that the absence of strong unimaginability cannot be ruled out in principle. Therefore, a situation is possible in which the initial apodicticity claim is in fact falsified. However, as I have already indicated, there is another way to look at things: Instead of taking the eyeball-TE to refute the initial apodicticity-claim, it could also be taken to show that there is something wrong with the conception of apodicticity ${ }_{1}$. I believe that the second option is indeed the correct one. Let us turn to that now.

Phenomenologically construed, the term "apodicticity" denotes a special quality of fulfillment, or, to put it differently, a special kind of Evidenz. This is crucial for the following reason: To speak of apodictic Evidenz (rather than, for instance, of apodictic truth) is to emphasize the fact that apodicticity is always given as the content of a concrete experience. Apodicticity is not detached from conscious life. It is rather a special kind of experience that, consequently, fits into the general structure of intentionality. This, however, is of crucial importance for the following reason: One of the most fundamental characteristics of conscious life is its temporality. All intentional acts have a position in the constant flux of internal time. They are related to each other in terms of "before", "after" and "at the same time as". And it is due to the unceasing flow of time that present acts constantly sink into the past from where they are restored either by retention or by acts of reflection and recollection.

On a phenomenological view, temporality is the most fundamental of all intentional accomplishments. The reason for this is that intentional acts are the exclusive medium of all of our cognitive practices. Whichever way we choose to make cognitive contact with ourselves or with our natural surroundings, it is always through intentional acts that we deal with what is accessible to us. However, if it is true that intentional acts are the sole medium of our cognitive practices, and if it is furthermore correct that intentional acts are necessarily 
embedded in the temporal structure of consciousness, then this tells us something utterly important about the very concept of validity. To make a long story short: If validity claims, how high their degree of certainty ever may be, are necessarily given in acts which themselves are temporarily constituted, then the concept of validity itself cannot be a-, extra- or supra-temporal. Phenomenologically construed, validity claims can be, at best, omnitemporal. "Omnitemporality" means that, ideally, validity claims can be reproduced at any point in time. However, this does not put them beyond time. For reasons we have just considered, "omnitemporality [...] nevertheless is a mode of temporality" (Husserl 1973, 261).

It goes without saying that the notion of omnitemporality has severe consequences for a conception of apodicticity that conforms to the standards of phenomenology. Insofar as it implies the idea of total security from error, apodicticity ${ }_{1}$ clearly depends on an a-temporal ideal of validity and therefore goes beyond what is acceptable from a phenomenological viewpoint. Apodicticity ${ }_{2}$, on the other hand, replaces the ideal of a-temporality with the ideal of omnitemporality. Apodicticity in this latter, phenomenological sense merely implies the claim that something, as a matter of descriptive fact, is unimaginable in a strong sense of the word. But, phenomenologically construed, there is no comprehensible reason to claim that this will be the case forever and in all possible worlds. Accordingly, what the eyeball-TE does is to highlight this crucial difference between apodicticity ${ }_{1}$ and apodicticity ${ }_{2}$.

Yet, isn't that still too much of a concession? One could find fault with the following: If apodicticity $_{2}$ does not contain the ideal of total security from error, then this is just another way of saying that apodictic judgment are in fact fallible. However, doesn't the distinction between the realm of the empirical and the realm of the essential collapse if this concession is made? Remember why we came to the conclusion that the former is contingent: Judgments about empirical matters of fact are contingent insofar as they are what they are, but could imagined differently. Yet, since, as we have seen, a falsification even of apodictic judgments cannot be ruled out, judgments about essences don't seem to be any better off. When it comes to their contingency, the difference between essential and empirical judgments appears to be, at best, a difference in degree, but certainly not in kind. But since this difference is crucial for the phenomenological project as such, this result is disastrous.

I believe that this conclusion is simply wrong. It rests on a conflation of two interrelated, but nevertheless nonequivalent conceptions. Here is what I mean: Empirical matters of fact are, as I have pointed out, contingent because they could be imagined differently. In the case of the law of gravity, for instance, the imagination of a concrete counterexample is clearly possible. We have no problem whatsoever with imagining a concrete $x$ (a mug that falls towards the center of the earth) being a concrete y (a mug that floats away from the ground without the application of external force). To say that the same holds true of apodictic judgments about essential laws, however, implies that "to imagine a concrete $\mathrm{x}$ being a concrete $y$ " means the same thing as "to imagine a concrete $x$ being not the case". Let me explain.

The only concession that apodicticity ${ }_{2}$ makes is that an instance of apodictic Evidenz could be possibly falsified by the absence of the experience of strong imaginability. Yet, this is not tantamount to the actual imagination of the experiences that we would have in this situation. The eyeball-TE illustrates this difference nicely: It may be possible that we perceive with a sphere of hundred eyeballs. And it may be the case that an apodictic judgment concerning the law of givenness in adumbrations is falsified in this situation. But, as a matter of descriptive fact, it is impossible to imagine the experiences that a subject for whom this law is actually falsified would have. Hence, there is a describable difference between the contingency that is characteristic for the empirical sphere and the peculiar kind of fallibility that is characteristic for the sphere of the essential. 


\section{Concluding Remarks about Fulfillment}

As I have tried to show, an evaluation of the eyeball-TE leads to a twofold result: On the one hand, as we have seen in the previous section, the TE is successful in the sense that it indicates a possible tension within the phenomenological framework. It thus forces phenomenologists to specify their conception of apodictic Evidenz. Yet, on the other hand, the TE fails if it is understood to be a straightforward refutation of the phenomenological position. If my considerations are sound, the TE fails on this level due to a confusion of what I have called first-person-imagination and second-person-imagination, respectively. However, there is one last question that I would like to consider: Why is it that the distinction between first-person-imagination and second-person-imagination matters, anyway? I believe that an important moral about TEs can be drawn from an answer to this question.

A distinction crucial to phenomenological epistemology is the one between empty and fulfilled intentions. Consider the following example from the sphere of ordinary perceptions: Suppose that I am thinking of going to the department kitchenette to make myself a mug of coffee. Part of this intend is a conception of my favorite red mug without actually seeing it. As long as I am still in the office, and the mug is still in the kitchenette, my intentions towards the mug are empty; I am merely thinking of it. Additionally, there are a number of expectations that come with these empty intentions: I expect the mug to have a certain appearance; I expect it to feel in a certain way; I expect it to hold a certain amount of coffee; I expect it to be where I always put it; and so on and so on. Since it is my favorite mug and since I have used it many, many times, I am pretty sure that I know all these things. However, I also know that sometimes even the most certain expectations can go wrong. Therefore, my reliance on empty intuiting (such as merely thinking about something or inferring something from something else) depends on the possibility to transform empty intuitions into fulfilled intuitions. In example of the mug, this transformation simply consists in going to the kitchenette and check. If I did, my empty intentions towards the mug would be fulfilled by its direct, unmediated givenness.

What this example illustrates is this: Fulfillment is an "Idea (in the Kantian sense)" (Husserl 1983,342 ) that regulates all those intentional references in which the respective things are not directly given. Or, to put it differently: The immediate givenness of that which is intended is the ideal limiting case to which all cases of mediate reference adhere. To be sure, this does not mean that knowledge and successful justification necessarily require the actual immediate givenness of the respective object or state of affairs. What it in fact means, however, is that "[k]nowing is [...] nothing other than actual or potential insight" (Husserl $2008,13)$. Hence, the possibility for a judgment to yield rational weight at all depends on the possibility of a situation in which the intended object or state of affairs could be directly given. ${ }^{12}$

The important point is that all this is true not only of ordinary perceptions. It is also and especially true of the imagination and thus of thought experimental reasoning. Consider the judgment that the law of gravity is contingent because I could imagine a concrete counterexample. Here, the intention towards a counterexample could be easily fulfilled by the sensory imagination of a mug that floats away from the ground without the application of external force. I could report the imaginative experiences that I would have in this situation from the perspective of first-person-imagination. In the case of a subject that perceives with hundred eyeballs, however, no such thing is possible. As a matter of descriptive fact, the intention towards a situation in which the law of givenness of adumbrations would be falsified remains empty. To be sure, it is possible to imagine the situation from the external viewpoint of third-person-imagination. But it is impossible to

\footnotetext{
${ }^{12}$ I give a more detailed account on this issue in my 2012.
} 
carry through to the ideal of immediate givenness, i.e. to the imagination of the actual experiences that a subject would have in this situation. And it is for this reason that the eyeball-TE lacks rational weight if it is intended as a straightforward refutation of the phenomenological law of givenness in adumbrations. ${ }^{13}$

\section{Literature}

Dennett, D.: Consciousness Explained. Boston 1991.

Fehige, Y. J. H. \& Wiltsche, H. A.: The Body, Thought Experiments, and Phenomenology. In: Brown, J. R., Frappier, M. \& Meynell, L. (eds.): Thought Experiments in Science, Philosophy, and the Arts. London 2012. pp. 69-89 (Routledge Studies in the Philosophy of Science; forthcoming).

Froese, T. \& Gallagher, S.: Phenomenology and Artificial Life: Toward a Technological Supplementation of Phenomenological Methodology. In: Husserl Studies 26, 2010, pp. 83106.

Gendler, T. S.: Thought Experiment. On the Power and Limits of Imaginary Cases. New York 2000.

Gendler, T. S. \& Hawthorne, J. (eds.): Conceivability and Possibility. Oxford 2002.

Gurwitsch, A.: The Field of Consciousness. Theme, Thematic Field and Margin. ed. by Zaner, R., Dordrecht 2010.

Häggqvist, S.: A Model for Thought Experiments. In: Canadian Journal of Philosophy 39/1, 2009, pp. 55-76.

Hopp, W.: Perception and Knowledge. A Phenomenological Account. Cambridge 2011.

Husserl, E.: Cartesian Meditations. An Introduction to Phenomenology. trans. by Cairns, D., The Hague 1960.

Husserl, E.: Experience and Judgment. Investigations in a Genealogy of Logic. trans. by Churchill, J. S. \& Ameriks, K., Evanston 1973.

Husserl, E.: Ideas Pertaining to a Pure Phenomenology and Phenomenological Philosophy. First Book. General Introduction to a Pure Phenomenology. trans. by Kersten, F., The Hague 1983.

Husserl, E.: Thing and Space. Lectures of 1907. trans. by Rojcewicz, R., Dordrecht et al. 1997.

Husserl, E.: Introduction to Logic and Theory of Knowledge. Lecture 1906/1907. trans. by Hill, C. O., Dordrecht 2008.

Mohanty, J. N.: The Method of Imaginative Variation in Phenomenology. In: Horowitz, T. \& Massey, G. J. (eds.): Thought Experiments in Science and Philosophy. Savage 1991, pp. 261272.

Schmid, H. B.: Apodictic Evidence. In: Husserl Studies 17, 2001, pp. 217-237.

Smith, D. W.: Husserl. New York 2007.

\footnotetext{
${ }^{13}$ This paper was presented at a research colloquium at the University of Graz, Austria, and at the annual meeting of the Nordic Society for Phenomenology (NOSP) in Oslo, Norway. I wish to thank the respective audiences and especially Michael Wallner for a number of fruitful comments. I also want to say thanks to the Austrian Science Fund (FWF) for their generous support without which my ongoing research on TEs would not be possible (project number: J 3114).
} 
Strawson, P. F.: Perception and its Objects. In: Noë, A. \& Thompson, E. (eds.): Vision and Mind. Selected Readings in the Philosophy of Perception. Cambridge 2002, pp. 91-110.

Vendler, Z.: The Matter of Minds. Oxford 1984.

Weinberg, J. M., Nichols, S. \& Stich, S.: Normativity and Epistemic Intuitions. In: Philosophical Topics 29, 2001, pp. 429-460.

Wilkes, K.: Real People. Personal Identity Without Thought Experiments. Oxford 1988.

Wiltsche, H. A.: What Is Wrong With Husserl's Scientific Anti-Realism? In: Inquiry 55/2, 2012, pp. $105-130$ 